soil properties down the profile, intermittent movement of water, interaction between the soil and the nutrients and so forth. Any model of leaching must deal both with water movement through the soil and with the interaction of the nutrients that it carries with the soil: at present there is insufficient knowledge to allow a model to be built up on a purely theoretical basis. The attempt to formulate theoretical models does, however, have the added advantage of guiding research into the most fruitful areas.

An alternative approach is to use numerical computer solutions to find the parameters necessary in the predictive equations. This approach can be used where a sufficient body of practical data exists for a particular situation. Much more information is needed to extend such models to the more general cases and the remaining speakers provided instances of how this information has been collected in practice. Information on both water and salt movement through soil was provided by lysimeter studies discussed by Mr A. J. Low (ICI, Jealott's Hill), Mr E. A. Garwood (Grassland Research Institute, Hurley) and by Mr J. Webber (ADAS, Leeds). Relevant information can also come from measurements of nutrient losses from agricultural topsoils as was reported by $\mathrm{Mr}$ R. J. B. Williams and $\mathrm{Dr}$ J. Bolton (Rothamsted Experimental Station).

The lysimeter studies emphasize that water movement through the profile is related to crop growth. Transpiration by a healthy crop much reduces the amount of water that is available for leaching. The situation is more complex, however, in that, for example, clover delays the onset of leaching longer than a grass canopy although the amount of water transpired is less. The Hurley workers have found another complexity of the water/crop interaction. Here nutrient loss in winter was related to the amount of water that had been available to the crop during the previous growing season. This was because drought restricted nutrient uptake (particularly nitrate) and so left greater residues in the soil for leaching during the following winter.

Nutrient losses from the topsoil of the Rothamsted experimental field Broadbalk during the past 100 years were presented. Here, a knowledge of the relationships between ions in the soil solution (primarily dictated by $p \mathrm{H}$ and $\mathrm{CO}_{2}$ partial pressure) allows detailed information on calcium loss to be used to predict total nutrient losses.

Perhaps the most practical suggestion which emerged from the meeting was from Mr Williams who suggested that losses of nitrate from the topsoil would be minimized if soil could be engineered to store more water and hence more nutrients within the rooting zone.
FRESHWATER FISH

\section{Irish Studies on Gudgeon}

from a Correspondent

A RECENT study by Michael Kennedy and Patrick Fitzmaurice on some aspects of the biology of the gudgeon in Irish waters (J. Fish Biol., 4, 425; 1972) has made an important contribution to the body of knowledge about the life history of this fish. The gudgeon is a small bottom-living member of the carp family which is widely distributed in Europe. Partly because of its size (it rarely grows to a length greater than $20 \mathrm{~cm}$ ) it is not the subject of great interest to inland fisheries, and few attempts have previously been made to study its life history or its relations with more economically important fishes.

The gudgeon is not a native fish in Ireland, having been introduced at some unrecorded date, and its distribution, although not well known, seems to be patchy. It is known to be common in the catchments of the Erne, the Boyne, the Cork Blackwater, the Lee and the Shannon, although it occurs in small numbers elsewhere, including still waters. Kennedy and Fitzmaurice studied samples from several reservoirs, lakes and streams, including trout streams in Co. Cork, and from the Grand Canal in Co. Dublin.

This is the first study to have been made of Irish gudgeon and the authors find general agreement between the life style of the species that is published in studies of European and British gudgeon, although one of the most important, B. Bernet's work (Ann. Sta. Cent. Hydrobiol. Appl., 8, 129; 1960), is not mentioned.

The preferred habitat of the gudgeon seems to be gravel-bottomed rivers and streams, although when reservoirs were constructed on the River Lee, a great increase in the gudgeon population was observed for a short period, followed by a return to a more normal density. Its food varies considerably with its habitat; young gudgeon in still waters feed on Cladocera and copepods to a much greater extent than those living in rivers. No matter what the environment, the principal foods eaten by the gudgeon are chironomid and trichopteran larvae, and ephemeropteran nymphs. In some localities large numbers of molluscs are eaten, in others filamentous algae are consumed. In general terms the gudgeon in Ireland is an adaptable fish feeding on, or close to, the bottom on whatever invertebrates are locally abundant, and even capable of utilizing algae as food (possibly in the absence of adequate animal prey).

Although the gudgeon is not regarded as a noticeable fish for inland fisheries purposes its potential as prey for, and competitor with, more valuable fishes has to be assessed. It is this aspect which clearly interests the staff of the Inland Fisheries Trust, and which has led to a series of valuable studies of the

\title{
Vesicular Stomatitis Virus Pseudotypes
}

IN next Wednesday's Nature New Biology (November 22) Zavada and his colleagues describe a new and ingenious technique they have developed to search for putative human cancer viruses. Zavada has found that when vesicular stomatitis virus (VSV), an enveloped RNA virus which is not oncogenic, infects chick cells previously infected by the oncogenic virus avian myeloblastosis virus (AMV) some of the progeny particles released are pseudotypes-VSV genomes enclosed in an envelope which has AMV antigens. These pseudotypes can be neutralized by anti-AMV antisera but not by antiVSV antisera and they have the host range and interference specificities of AMV, not VSV.

When wild-type VSV is used to infect cells already infected with AMV some wild-type VSV particles are produced in addition to VSV(AMV) pseudotypes. To eliminate these contaminating wildtype VSV particles Zavada selected mutant strains of VSV the virus particles of which are more thermolabile than the wild-type particles. He then infected with these mutant VSV cells infected with AMV and simply by heat- ing eliminated progeny VSV particles enclosed in VSV envelopes. The pseudotypes, VSV genomes in AMV envelopes, survived because the temperature sensitive lesion in the mutant VSV strains is located in a component of the VSV envelope.

Zavada and his colleagues have, as they explain in their second report, infected cultures of human mammary carcinoma cells, $\mathrm{HeLa}$ cells and other human cells with such mutant VSV and screened for progeny particles which are not thermolabile and lack VSV neutralization antigens. Only the human mammary carcinoma cells yielded such particles and the possibility remains that these particles are pseudotypes with VSV genomes in envelopes the antigens of which are specified by a putative human tumour virus resident in the mammary carcinoma cells.

Of course this is not the only possible explanation of the origin of these VSV pseudotypes but it has not yet been excluded and the method seems to provide a new approach to the problem of screening human cancer cells for traces of human cancer viruses. 